\title{
Price Dynamics under the Information-Based Dealer Model
}

\author{
Kenta Fukuda, Kaichi Kondo, Hideyuki Takada \\ Department of Information Sciences, Toho University, Chiba, Japan \\ Email: 6519008f@st.toho-u.jp,6519004k@st.toho-u.jp, hideyuki.takada@is.sci.toho-u.ac.jp
}

How to cite this paper: Fukuda, K., Kondo, K. and Takada, H. (2019) Price Dynamics under the Information-Based Dealer Model. Journal of Mathematical Finance, 9, 726-746.

https://doi.org/10.4236/jmf.2019.94037

Received: September 30, 2019

Accepted: November 11, 2019

Published: November 14, 2019

Copyright $\odot 2019$ by author(s) and Scientific Research Publishing Inc. This work is licensed under the Creative Commons Attribution International License (CC BY 4.0). http://creativecommons.org/licenses/by/4.0/

\begin{abstract}
We investigate the stochastic behavior of the transaction price process formed by the information-based dealer model. We extend two-agent model of Nadi Serhan Aydin to multi-agent case to see the effect of the market size and noise correlations. Applying the Monte Carlo method, our numerical findings are summarized as 1) volatility of the transaction price depends on both of the noise correlation and the market size, 2) the price process has a long term memory and its Hurst extend depends on both of the noise correlation and the market size.
\end{abstract}

\section{Keywords}

Information-Based Approach, Dealer Model, Long Term Memory

\section{Introduction}

Market participants hope to know the true value of financial assets based on their available information. To take government bond as an example, the issuance price can be easily observed in a primary market as a unique value, and also redemption price is predetermined. However, in the secondary market, it is not easy to evaluate uniquely the theoretical price of the government bond due to several uncertainties. There is a wide range of view or estimation in the market (thus the sell and buy orders can meet) and such a diversity will grow over time. Since the redemption price is predetermined, the diversity will gradually shrink as time passes, and finally converges to the face value at maturity. In a nutshell, market information plays an essential role in asset pricing.

In that spirit, Brody et al. [1] proposed so-called information-based approach. Fundamental idea is that when a market participant decides to trade an asset, the decision is made in accordance with the information available to the market par- 
ticipant concerning the likely future cash flows associated with the asset. Thus the approach gives prime importance to the hypothesis that the change in the information available to the market participants will have an effect on the price at which they are willing to trade. In order to represent such information flying over the market, bridge processes are utilized to model non-separable noise part. The framework has been organized at full length by Macrina [2] and Hoyle [3], and extended to several directions by Rutkowski \& Yu [4], Brody et al. [5], Brody et al. [6], and Macrina \& Parbhoo [7]. In this paper, we will briefly introduce the information based approach in the next chapter.

Brody et al. [5] also proposed that the price determination process between two agents trading in the secondary market can be described by the information based approach. In this application, it is assumed that different agents have access to different information and each agent is unaware of each other's theoretical prices. Typical structure is that the learning process is incorporated in this framework, i.e., when a trade executes, each trader learns the price of the other, and as a consequence can back out the value of corresponding information, which would help to calculate better price.

Nadi Serhan Aydin [8] extended the agent-based model of [5] more explicitly so as to represent realistic learning process. That is, the delayed identification of counterparty's information due to the assumption that the agents are not allowed to observe another agent's theoretical price, unless the trade executed. Therefore, the agent can utilize her counterparty's intermittent information garnered at every execution as well as her own continuously observed information in order to evaluate better price for the profit. Both [5] and [8] considered independent noise for simplicity.

In this paper, we will pay attention to the correlation for noise process, and focus on the numerical analysis to understand the stochastic behavior of the transaction price under multi-agent setting. The noise correlation can be understood to represent the tendency to receive biased information for agents. We found numerically that 1 ) the realized volatility of the transaction price depends on the correlation of the noise, and 2) the transaction price process has a long term memory, i.e., its Hurst exponent value exceeds 0.5. As some empirical studies support the fractal market model, our agent-based model incorporating the learning process would be a prospective candidate for multi-agent dealer model.

The paper is organized as follows. In Section 2, we survey the information-based approach to emphasize that the pricing formula can be seen the deterministic increasing function of so-called market information. In Section 3, we propose an information updating and extend the framework so as to analyze multi-agents dealer model. Monte Carlo simulation method is discussed in Section 4 and numerical experiences are illustrated to grasp the stochastic behavior of the transaction price. Taking into account the fact that the learning procedure is built in our proposed framework, we numerically study in Section 5 about the presence or absence of the long term memory. We conclude in Section 6. 


\section{Information-Based Approach}

We would briefly survey the Information-based approach introduced by Brody, Hughston and Macrina (BHM in short) according to [1] [2] [4] and [5]. BHM focuses on the aspect that the revelation of a new piece of information moves the market sentiment and then causes the price change. Here the information in mind can include both true, so valuable information, and false, so valueless information. Unlike most other financial modelings, BHM first specifies a flow of market information available to market participants in the following way: every tradable asset is characterized by its own cash flows and the market information is assumed to have the form of a superposition of a signal component associated with each cash flow and an independent noise component. These two components are not separable and then fluctuation of the noise determines the quality of the market information. In general, cash flows may depend on some random factors representing financial environment or individual condition of company or country. The next step of BHM is, as common to other standard frameworks, to take expectation of the discounted cash flow with respect to pricing measure conditional on the market information. Therefore, whether the price process is adapted to the filtration generated by the market information is not trivial by its nature.

Original framework of BHM is mainly devoted to valuation of the financial instruments, but not restricted to it. For example, it can be applied to 1) Information disparity and statistical arbitrage [5], 2) Price formation in inhomogeneous markets [5] [8], and also to 3) Insider trading [6]. This study is classified into the second topic 2), concerned with the transaction price formation under agent-based inhomogeneous market model. We first survey single agent case that is originally designed to asset pricing, and then generalize to multi-agent case in the subsequent section.

Uncertainty is modeled by a probability space $(\Omega, \mathcal{F}, \mathbb{P})$ on which a filtration $\left(\mathcal{F}_{t}\right)_{0 \leq t<\infty}$ describing market information flow will be specially constructed. The probability measure $\mathbb{P}$ serve as the pricing measure chosen by the market. Let $B_{t}$ be a standard Brownian motion on $(\Omega, \mathcal{F}, \mathbb{P})$. Fix the terminal time $T<\infty$ and introduce standard Brownian bridge on the interval $[0, T]$ given by $\beta_{t} \stackrel{\text { def }}{=} B_{t}-\frac{t}{T} B_{T}$. We can easily see $\beta_{t} \sim N(0, t(T-t) / T)$ and $\mathbb{E}\left[\beta_{s} \beta_{t}\right]=s(T-t) / T$ for $0 \leq s \leq t \leq T$.

\subsection{Information Process}

Although the BHM framework can handle multiple cashflows and some structures referred as $X$-factor analysis, hereafter for simplicity, we consider single cashflow representing principal repayment of a defaultable discount bond. Let $X_{T}$ be an integrable random variable representing payoff at maturity $T$ of defaultable bond. The market information about the cash flow is assumed to have the following form:

$$
\xi_{t} \stackrel{\text { def }}{=} \sigma t X_{T}+\beta_{t}, \sigma \in \mathbb{R}_{+},
$$


where $X_{T}$ and $\beta_{t}$ are mutually independent under $\mathbb{P}$. The positive parameter $\sigma$ is called information flow rate (see [5]) because, as we will see later the trajectory of the price process, it controls the rate at which the true value of $X_{T}$ is revealed as time progresses. So we can also translate the parameter $\sigma$ as a research skill of the market participant because she doesn't have direct access to $X_{T}$. The reason why the BHM framework employs the Brownian bridge is suitably described in Section 2.1 of [8], to quote, There is normally little or no rumor about an asset's future value when there is a significant time frame until its maturity, the beliefs are most diverse around midway through the life time of the asset when the rumors intensify, there is a growing consensus, as the asset approaches its maturity, on how things will turn out, and, finally, the true value becomes known.

Now we can construct the filtration $\mathcal{F}_{t}$ as the information generated by the process $\xi_{t}$;

$$
\mathcal{F}_{t} \stackrel{\text { def }}{=} \sigma\left(\xi_{u} ; 0 \leq u \leq t \leq T\right) .
$$

Thus we see that $X_{T}$ is $\mathcal{F}_{T}$-measurable but not $\mathcal{F}_{t}$-measurable for any $t<T$. We must note that the notation $\sigma\left(\xi_{t}\right)$ denotes the smallest $\sigma$-algebra generated by $\left(\xi_{u}\right)_{u \leq t}$ and thereby different from information flow rate parameter $\sigma$.

\subsection{Objective Value of the Asset}

We can evaluate, based on the market information $\mathcal{F}_{t}$, the theoretical price $S_{t}$ of defalutable discount bond with maturity $T$ as

$$
S_{t}=\mathrm{e}^{-r(T-t)} \mathbb{E}_{\mathbb{P}}\left[X_{T} \mid \mathcal{F}_{t}\right] .
$$

For simplicity, we assume that risk-free interest rate $r$ is zero. Furthermore as [2] [4] [8] and Brody et al. [9] mentioned, it is known that the process $\left(\xi_{t}\right)$ is Markov with respect to $\left(\mathcal{F}_{t}\right)_{t \geq 0}$. Hence, $(1)$ is rewritten by

$$
S_{t}=\mathbb{E}_{\mathbb{P}}\left[X_{T} \mid \xi_{t}\right] .
$$

If we introduce the conditional probability density $\pi_{t}(x)$ for $X_{T}$ given $\mathcal{F}_{t}$

$$
\pi_{t}(x) \stackrel{\text { def }}{=} \frac{\mathrm{d}}{\mathrm{d} x} \mathbb{P}\left(X_{T} \leq x \mid \xi_{t}\right),
$$

then (2) can be expressed as

$$
S_{t}=\int_{-\infty}^{\infty} x \pi_{t}(x) \mathrm{d} x
$$

Let $p(x)$ denotes the a priori probability density of $X_{T}$ known as an initial condition and then via Bayes formula, conditional density $\pi_{t}(x)$ can be derived as a posteriori probability;

$$
\pi_{t}(x)=\frac{p(x) p\left(\xi_{t} \mid X_{T}=x\right)}{\int_{-\infty}^{\infty} p(y) p\left(\xi_{t} \mid X_{T}=y\right) \mathrm{d} y} .
$$


Next, we need to know the likelihood $p\left(\xi_{t} \mid X_{T}=x\right)$ to complete the calculation. Given $X_{T}=x$, the distribution of $\xi_{t}=\sigma t X_{T}+\beta_{t}$ is determined by its mean and variance

$$
\mathbb{E}\left[\xi_{t} \mid X_{T}=x\right]=\sigma t x, \operatorname{Var}\left[\xi_{t} \mid X_{T}=x\right]=t \kappa_{t}^{-1}, \kappa_{t}:=T /(T-t) .
$$

Hence we have

$$
p\left(\xi_{t} \mid X_{T}=x\right)=\frac{1}{\sqrt{2 \pi} \sqrt{t \kappa_{t}^{-1}}} \exp \left(-\frac{\left(\xi_{t}-\sigma t x\right)^{2}}{2 t \kappa_{t}^{-1}}\right)
$$

and then by inserting (4) to (3) yields

$$
\pi_{t}(x)=\frac{p(x) \exp \left(\kappa_{t}\left(\sigma x \xi_{t}-\frac{1}{2} \sigma^{2} x^{2} t\right)\right)}{\int_{-\infty}^{\infty} p(y) \exp \left(\kappa_{t}\left(\sigma y \xi_{t}-\frac{1}{2} \sigma^{2} y^{2} t\right)\right) \mathrm{d} y} .
$$

The market participants can use this formula for best-estimate of the future cash flow $X_{T}$ based on the available information $\xi_{t}$ at time $t$.

Finally we emphasize that the pricing formula

$$
S_{t}=\frac{\int_{-\infty}^{\infty} x p(x) \exp \left(\kappa_{t}\left(\sigma x \xi_{t}-\frac{1}{2} \sigma^{2} x^{2} t\right)\right) \mathrm{d} x}{\int_{-\infty}^{\infty} p(y) \exp \left(\kappa_{t}\left(\sigma y \xi_{t}-\frac{1}{2} \sigma^{2} y^{2} t\right)\right) \mathrm{d} y}
$$

can be seen as a deterministic function $\mathbb{S}: \mathbb{R} \rightarrow \mathbb{R}$ of $\xi_{t}$ for each time $t$, with predetermined constant parameter $\sigma$, i.e., $S_{t}=\mathbb{S}\left(\xi_{t} ; \sigma, t\right)$.

\section{Agent-Based Inhomogeneous Market Model}

In this section, we extend the single-agent case summarized in the previous section to the multi-agent case. We consider $N \geq 2$ individual market participants (agents) who want to sell and buy one asset in the market. For notational convenience, we write $\mathcal{N}:=\{1,2, \cdots, N\}$ for the set of all agents. All the agents are supposed to act as market maker in quote driven market enabling them to post the bid and ask price that they are willing to trade. Main difference from the single-agent case is to expand our interest to the situation where different agents have access to different information. Brody et al. [5] introduced two agents and studied the dynamics of transaction price formed by their trading behaviors as a consequence of the assumption that the two agents will work out different objective prices based on their own information garnered. One important structure of this framework is that when a trade occurs, each agent observes the price of the other and then can back out on the value of the corresponding information. Furthermore N. Serhan Aydin [8] extended the price formation framework of [5] so as to consider the elapsed time from the execution that would make asymmetry of obtained information. This standpoint is compatible with the realistic assumption such that the agents are attentive and infer their counterpart's posterior density of $X_{T}$ from their quote prices at the execution. This additional 
information will supplement her own information to reach more accurately and more quickly to the true value of $X_{T}$.

The above two existing research assumed that the correlation of noise process is zero, however we attach great importance to the dependent noise, which will be apparent numerically, have an impact on the dynamics of the transaction price. Besides, we present one simple way to extend the framework from two-agents case to general $N$-agents case. Then our concern would be that what will happen if the number of agents increases, and also what will be invariant even if the number of agents increases. We assume that $N$ agents behave under risk-neutral, so each doesn't care about the utility function.

Let $\xi_{t}^{n}$ denote the market information for the agent $n$ at time $t$ and assume below.

Assumption 1. The information flow for agent $n$ has the form of

$$
\xi_{t} \stackrel{\text { def }}{=} \sigma_{n} t X_{T}+\beta_{t}^{n}, n \in \mathcal{N},
$$

where $\beta_{t}^{n}(n \in \mathcal{N})$ are standard Brownian bridges on $(\Omega, \mathcal{F}, \mathbb{P})$ and are all independent of $X_{T}$, while satisfies $\operatorname{Corr}\left(\beta_{t}^{n}, \beta_{t}^{m}\right)=\rho \geq 0$ for all $n, m \in \mathcal{N}$. Each agent $n$ is assumed to know the value of $\rho$ and the median $\bar{\sigma}$ of all agents' information flow rate. Moreover, every agents have a knowledge of prior distribution $p(x)$ of $X_{T}$ and are assumed to common for all agents.

Remark 1. Assumption that every agent has a knowledge of the median $\bar{\sigma}$ of all agents is compatible with the $N=2$ case of [5] [8] and Kondo et al. [10] assuming that the agents know exact value of the information flow rate for each other.

\subsection{Transaction Price}

The agent $n$ works out her objective price (viz. her own theoretical price) $S_{t}^{n}$ at time $t$ based on her available information. Here the available information would be a combination of her own $\xi_{t}^{n}$ and some supplemental information that will be formulated explicitly later on. Every agent is unaware of each other's objective prices, but can observe transaction price determined as the following. Moments before the sequential auction time $t$, each agent submits to the central-planner her theoretical price at which she is willing to trade. To keep our framework comparable to that of [5] and [8], we assume that the executions are always done with one unit of asset, and bid-ask spread is set to 0 . Matching rule of $\mathrm{N}$-agent market is assumed as follows.

Assumption 2. The transaction price at time $t$, denoted by $S_{t}^{*}$, is determined by the central clearing party as the median of all the submitted prices.

Thus, if $N=2 k$ or $N=2 k+1$, then just $2 k$ agents execute at the same instant. We note that every quote prices are blind to every agent while on the other hand the central clearing party can see all these quotes. And the central clearing party, complying with the predetermined matching rule as above, decides not only whether the trade occurs or not and the transaction price, but also who sells and who buys the asset. 


\subsection{Information Structure}

We extend the pricing formula (5) so as to reflect the supplemental information, i.e., arguments of the pricing formula would be twofold; continuously observable market information of her own, and supplemental information obtained by recent transaction. So, let the pricing formula for the agent $n$ have a tentative form of binary function ${ }^{1}$ such as $S_{t}^{n}=\mathbb{S}\left(\xi_{t}^{n}, \xi_{s_{t}}^{n *}\right)$, where $\xi_{s_{t}}^{n *}$ denotes the supplemental information that might be obtained by recent transaction regardless of whether she could be involved or not. It is worth pointing out that $\xi_{s_{t}}^{n *}$ does not indicate some specific agent's market information, but indicates the aggregate information that would be interpolated for $n$ in order to work out her objective price agreeing on the transaction price. Here, $s_{t}$ stands for the latest time before time $t$ such that the recent transaction occurred. In our matching rule, the supplemental information is assumed to be renewed at each transaction; $s_{t}$ runs one tick behind the business time $t$.

When the agent $n$ wants to calculate her own theoretical price at time $t$, she can utilize the aggregate information $\xi_{s_{t}}^{n *}$ that might be circulated a moment before the recent transaction. Therefore, all the information accessible for the agent $n$ at time $t$ is expressed as $\sigma\left(\xi_{t}^{n}\right) \vee \sigma\left(\xi_{s_{t}}^{n *}\right)$.

To conclude the subsection, we need to design the structure of supplemental information $\xi_{t}^{n *}$ in a consistent manner with (6). For each agent $n$, introducing Brownian bridge $\beta_{t}^{n *}$ that is independent of $X_{T}$, we can assume that the information $\xi_{t}^{n *}$ has the form of

$$
\xi_{t}^{n^{*}}=\sigma_{n^{*}} X_{T}+\beta_{t}^{n^{*}}, \sigma_{n^{*}} \in \mathbb{R}, \operatorname{Corr}\left(\beta_{t}^{n}, \beta_{t}^{n^{*}}\right)=\rho>0, \forall n \in \mathcal{N} .
$$

Remark 2. $\sigma_{n *}$ doesn't represent some specific agent's information flow rate.

\subsection{Objective Price in Agent-Based Model}

In this subsection, we will find the specific form of the pricing formula $\mathbb{S}: \mathbb{R}^{2} \rightarrow \mathbb{R}$ for multi-agent model tentatively introduced in the previous subsection. As before, let $\xi_{s_{t}}^{n *}$ denote the supplemental information that might be circulated on the market (but the agent $n$ couldn't catch) at the moment before the recent transaction. The agent $n$, regardless of whether she could get involved in the latest deal or not, calculates her own theoretical price at time $t$ given the market information as

$$
S_{t}^{n}=\mathbb{E}_{\mathbb{P}}\left[X_{T} \mid \sigma\left(\xi_{t}^{n}\right) \vee \sigma\left(\xi_{s_{t}}^{n *}\right)\right]=\int_{-\infty}^{\infty} x \bar{\pi}_{t}^{n}(x) \mathrm{d} x,
$$

where a posteriori probability of $n$ at time $t$ is represented by

$$
\bar{\pi}_{t}^{n}(x)=\frac{p(x) p\left(\xi_{t}^{n}, \xi_{s_{t}}^{n *} \mid X_{T}=x\right)}{\int_{-\infty}^{\infty} p(x) p\left(\xi_{t}^{n}, \xi_{s_{t}}^{n *} \mid X_{T}=x\right) \mathrm{d} x} .
$$

${ }^{1}$ By abuse of notation, we use same letter $\mathbb{S}$ for pricing formula to denote both the single-agent case i.e., (5) and the multi-agent case. 
By the definition of $\xi_{t}^{n}$ described in (6), under $X_{T}=x$,

$$
\xi_{t}^{n} \sim N\left(\sigma_{n} t x, t \kappa_{t}^{-1}\right) .
$$

On the other hand, our assumption (7) approves that the conditional distribution given $X_{T}=x$ is

$$
\xi_{t}^{n *} \sim N\left(\sigma_{n *} t x, t \kappa_{t}^{-1}\right)
$$

We can write

$$
\beta_{t}^{n}=\rho \beta_{t}^{n *}+\sqrt{1-\rho^{2}} \bar{\beta}_{t}, \beta_{t}^{n *} \Perp \bar{\beta}_{t},
$$

hence the correlation between different time can be calculated.

$$
\begin{aligned}
\operatorname{Corr}\left(\beta_{t}^{n}, \beta_{s_{t}}^{n *}\right) & =\frac{\operatorname{Cov}\left(\beta_{t}^{n}, \beta_{s_{t}}^{n *}\right)}{\sqrt{\operatorname{Var}\left(\beta_{t}^{n}\right)} \sqrt{\operatorname{Var}\left(\beta_{s_{t}}^{n *}\right)}} \\
& =\frac{\operatorname{Cov}\left(\rho \beta_{t}^{n *}+\sqrt{1-\rho^{2}} \bar{\beta}_{t}, \beta_{s_{t}}^{n *}\right)}{\sqrt{t \kappa_{t}^{-1}} \sqrt{s_{t} \kappa_{s_{t}}^{-1}}} \\
& =\rho \sqrt{\frac{s_{t} \kappa_{s_{t}}}{t \kappa_{t}}}=: \hat{\rho} .
\end{aligned}
$$

Since $\frac{s_{t}}{t}<1$ and $\frac{T-t}{T-s_{t}}<1$, it is easily seen that $|\hat{\rho}|<|\rho|$. Correlation between $\xi_{t}^{n}$ and $\xi_{s_{t}}^{n *}$ is also $\hat{\rho}$ and then the likelihood $\bar{p}\left(\xi_{t}^{n}\right)$ of the agent $n$ is given by

$$
\begin{aligned}
& \bar{p}\left(\xi_{t}^{n}\right) \stackrel{\text { def }}{=} p\left(\xi_{t}^{n}, \xi_{s_{t}}^{n *} \mid X_{T}=x\right) \\
& =\frac{1}{2 \pi \sqrt{s_{t} \kappa_{s_{t}}^{-1}} \sqrt{t \kappa_{t}^{-1}} \sqrt{1-\hat{\rho}^{2}}} \\
& \cdot \exp \left(-\frac{s_{t} \kappa_{s_{t}}^{-1}\left(\xi_{t}^{n}-\sigma_{n} x t\right)^{2}-2 \hat{\rho}\left(\xi_{t}^{n}-\sigma_{n} x t\right)\left(\xi_{s_{t}}^{n *}-\sigma_{n^{*}} x s_{t}\right)+t \kappa_{t}^{-1}\left(\xi_{s_{t}}^{n *}-\sigma_{n^{*}} x s_{t}\right)^{2}}{2\left(1-\hat{\rho}^{2}\right) t \kappa_{t}^{-1} s_{t} \kappa_{s_{t}}^{-1}}\right)
\end{aligned}
$$

Finally, we deduce objective price for the agent $n$ as follows.

$$
S_{t}^{n}=\frac{\int_{-\infty}^{\infty} x p(x) \exp \left[\kappa_{t}\left(\sigma_{n} \xi_{t}^{n} x-\frac{\sigma_{n}^{2} x^{2} t}{2}\right)+\rho s \kappa_{s}\left(-\left(\xi_{t}^{n} \frac{\sigma_{n^{*}}}{t}+\xi_{s}^{n *} \frac{\sigma_{n}}{s}\right) x+\sigma_{n} \sigma_{n *} x^{2}\right)+\kappa_{s}\left(\sigma_{n *} \xi_{s}^{n *} x-\frac{\sigma_{n^{*}}^{2} x^{2} s}{2}\right)\right] \mathrm{d} x}{\int_{-\infty}^{\infty} p(x) \exp \left[\kappa_{t}\left(\sigma_{n} \xi_{t}^{n} x-\frac{\sigma_{n}^{2} x^{2} t}{2}\right)+\rho s \kappa_{s}\left(-\left(\xi_{t}^{n} \frac{\sigma_{n^{*}}}{t}+\xi_{s}^{n *} \frac{\sigma_{n}}{s}\right) x+\sigma_{n} \sigma_{n *} x^{2}\right)+\kappa_{s}\left(\sigma_{n *} \xi_{s}^{n *} x-\frac{\sigma_{n *}^{2} x^{2} s}{2}\right)\right] \mathrm{d} x} .
$$

So we can see this formula as the binary function of $\xi_{t}^{n}$ and $\xi_{s_{t}}^{n *}$ given as $S_{t}^{n}=\mathbb{S}\left(\xi_{t}^{n}, \xi_{s_{t}}^{n *} ; \sigma_{n}, \sigma_{n^{*}}, t, s_{t}\right)$ with four parameters $\sigma_{n}, \sigma_{n^{*}}, t$ and $s_{t}$. This formula reflects the two kinds of information that appeared at different timing; the current information $\xi_{t}^{n}$, and the delayed information $\xi_{s_{t}}^{n *}$. We note that theoretical price $S_{t}^{n}$ depends not only on $\xi_{t}^{n}$, but also on $\xi_{s_{t}}^{n^{*}}$.

As a special case that simplifies the numerical calculation but remains to posses rich property, we consider Bernoulli distribution for a priori probability density $p(x)$ of $X_{T}$. More specifically, the payoff $X_{T}$ at maturity $T$ takes 
$x_{1}$ with probability $p_{1}$, while takes $x_{0} \in\left[0, x_{1}\right)$ with probability $p_{0}:=1-p_{1}$. Then we have, discretizing $x$ in (11),

$$
S_{t}^{n}=\frac{\sum_{i=0}^{1} x_{i} p_{i} \exp \left[\kappa_{t}\left(\sigma_{n} \xi_{t}^{n} x_{i}-\frac{\sigma_{n}^{2} x_{i}^{2} t}{2}\right)+\rho s \kappa_{s}\left(-\left(\xi_{t}^{n} \frac{\sigma_{n *}}{t}+\xi_{s}^{n *} \frac{\sigma_{n}}{s}\right) x_{i}+\sigma_{n} \sigma_{n *} x_{i}^{2}\right)+\kappa_{s}\left(\sigma_{*} \xi_{s}^{n *} x_{i}-\frac{\sigma_{n^{*}}^{2} x_{i}^{2} s}{2}\right)\right]}{\sum_{i=0}^{1} p_{i} \exp \left[\kappa_{t}\left(\sigma_{n} \xi_{t}^{n} x_{i}-\frac{\sigma_{n}^{2} x_{i}^{2} t}{2}\right)+\rho s \kappa_{s}\left(-\left(\xi_{t}^{n} \frac{\sigma_{n *}}{t}+\xi_{s}^{n *} \frac{\sigma_{n}}{s}\right) x_{i}+\sigma_{n} \sigma_{n *} x_{i}^{2}\right)+\kappa_{s}\left(\sigma_{n *} \xi_{s}^{n *} x_{i}-\frac{\sigma_{n *}^{2} x_{i}^{2} s}{2}\right)\right]}
$$

We note that from Assumption 1, each agent is assumed to know $\rho$ as built in the above pricing formula. Specially, setting $x_{0}=0, x_{1}=1, \rho=0$ and $\xi_{t}^{n}=\xi_{t}^{1}, \xi_{s}^{n^{*}}=\xi_{s}^{2}$ gives

$$
S_{t}^{1}=\frac{p_{1} \exp \left(\kappa_{t}\left(\sigma_{1} \xi_{t}^{1}-\frac{\sigma_{1}^{2} t}{2}\right)+\kappa_{s}\left(\sigma_{2} \xi_{s}^{2}-\frac{\sigma_{2}^{2} s}{2}\right)\right)}{p_{0}+p_{1} \exp \left(\kappa_{t}\left(\sigma_{1} \xi_{t}^{1}-\frac{\sigma_{1}^{2} t}{2}\right)+\kappa_{s}\left(\sigma_{2} \xi_{s}^{2}-\frac{\sigma_{2}^{2} s}{2}\right)\right)}
$$

which is introduced in [9] as credit risky bond with zero-recovery at default.

\section{Information Update}

We assume that the transaction price at time $t$ is announced as $S_{t}^{*}$ by the central-planner, however the exact value of all submitted theoretical prices are not notified. In case of $N=2$ and furthermore the bid-ask spread is known to every agent, as Serhan discussed in [8], each agent can see the price of orders submitted by her counterparty. Therefore, under the assumption that the two agents know counterparty's information flow rate, they can utilize both pieces of information at submission.

However, in case of $N>2$, the situation is slightly different; one can not identify who could sell. Hence we need to build some new model to represent information updating. When the agent $n$ wants to calculate her theoretical price, she hopes to utilize not only her own information $\xi_{t}^{n}$ but also some additional information $\xi_{s_{t}}^{n *}$ that might assist to evaluate a more accurate price. Such helpful information could be deduced from observable, that is, the transaction price $S_{S_{t}}^{*}$. Thus the problem is how to update the supplemental information $\xi_{s_{t}}^{n *}$ given $S_{s_{t}}^{*}$.

Suppose that the transaction has just been executed now, say time $t$. Then all the agents can observe the market price $S_{t}^{*}$ and next, each agent $n$ wants to find the pair $\left(\xi_{t}^{n *}, \sigma_{n^{*}}\right)$ that replicate $S_{t}^{*}$ at the moment after the execution based on her own pricing formula (11) or (12). However she can't know who could sell, hence she can't identify the value of the corresponding information flow rate $\sigma_{n^{*}}$. Instead, as we assumed in Assumption 1, she knows the median of the information flow rate of the other agents $\bar{\sigma}$. This quantity would be a substitute to replace $\sigma_{n *}$. We assume that $\sigma_{n^{*}}:=\bar{\sigma}$ for each $n$ in order to back out supplemental information $\xi_{t}^{n *}$ to replicate the transaction price. Therefore, she wants to find $\xi_{t}^{n *}$ satisfying

$$
S_{t}^{*}=\mathbb{S}\left(\xi_{t}^{n *}, \xi_{s_{t}}^{n *} ; \sigma_{n^{*}}, \sigma_{n^{*}}, t, S_{t}\right)
$$


for given $\xi_{s_{t}}^{n *}$, which was already derived in the same way at previous transaction and kept till now. The above non-linear equation can be solved numerically and derived $\xi_{t}^{n *}$ will be kept until the next submission of orders, say time $t^{\prime}$. At time $t^{\prime}$, agent $n$ will calculate her own theoretical price by

$$
S_{t^{\prime}}^{n}=\mathbb{S}\left(\xi_{t^{\prime}}^{n}, \xi_{t}^{n^{*}} ; \sigma_{n}, \sigma_{n^{*}}, t^{\prime}, t\right) .
$$

This cyclic procedure will continue to the maturity $T$.

\section{Monte Carlo Simulation}

In this section, we discuss the algorithm of Monte Carlo simulation for generating one scenario of the transaction price. We need realized value of $X_{T}$ and $N$ path $\left\{\beta_{t}^{n}\right\}_{t \in[0, T]}^{n \in \mathcal{N}}$ to complete single trial of the price trajectory simulation. In order to generate the trajectory of information flow for agent $n \in \mathcal{N}$

$$
\xi_{t}^{n}(\omega)=\sigma_{n} t X_{T}(\omega)+\beta_{t}^{n}(\omega)
$$

for randomly chosen $\omega \in \Omega$, we discretize the time interval $[0, T]$ into $K$ equal unite length $T / K$ and apply Euler-Maruyama scheme. Numerical stability of the Euler-Maruyama scheme is well established, for example, leader can refer Chapter 9 of Kloeden and Platen [11] and Chapter 3 of Paul Glasserman [12]. The algorithm generates one trajectory of the market price $S_{t}^{*}$ during $[0, T]$.

\section{Algorithm 1.}

Step 0. Set the all model parameters. i.e., $\sigma_{n}>0, \rho>0, x_{1}=1, x_{0} \in\left(0, x_{1}\right)$, $p_{0}, \quad p_{1}:=1-p_{0}$, the time grids $0=t_{0}<t_{1}<\cdots<t_{K}=T, t_{k+1}-t_{k}=T / K$ and $k=0, s_{t}=0$.

Step 1. Generate the realized value of $X_{T}(\omega)$ at the terminal T. And generate $N$ path of realized Brownian bridges $\left\{\beta_{t}^{n}(\omega), t \leq T\right\}_{n \in N}$ with correlation $\rho>0$.

Step 2. Set $k=k+1$. Calculate $S_{t}^{n}$ under $\sigma\left(\xi_{t}^{n}\right) \vee \sigma\left(\xi_{s_{t}}^{n *}\right)$ at time $t=t_{k}=t_{k-1}+T / K$.

Step 3. Given all the submitted prices $\left\{S_{t}^{n}\right\}_{n \in N}$, the central clearing party judges whether the trade executed and the transaction price. If the transaction succeeded, set $s_{t}=t$ and determine $S_{t}^{*}$. If nothing done, go to Step 5 .

Step 4. Observing the transaction price $S_{t}^{*}$, each agent update her own a posteriori distribution $\bar{\pi}_{t}^{n}(x)$ by finding the supplemental information $\xi_{t}^{n *}$ based on (13).

Step 5. Unless $t=T$, go to Step 2 and continue. If $t=T$, stop.

In order to generate a lot of scenarios, we need to repeat above loop (from Step 1. to Step 5.) for required number of times. Possible generalization that would increase the computational times is twofold; the number of partition $K$ and the number of the agents $N$. However, it is easy to see that both $K$ and $N$ increase the computational times linearly. It took approximately 0.2856 second for calculating just one scenario of transaction price for $K=250, N=100$ by Matlab 2018 with $3.4 \mathrm{GHz}$ Intel Core i7 processor and 32GB of RAM. In our numer- 
ical experiences, we set $T=1, p_{0}=0.2, p_{1}=0.8$ as fixed parameters, select $K=250$ or 1000 depending on the purposes, and the other parameters $\sigma_{n}, \rho$ are artificially changed to see how the price $S_{t}^{*}$ evolve. The correlation $\rho$ (between the pure noises) controls the degree to which the agents receive the biased information.

Remark 3. In general, it is known that

$$
\beta_{t}=B_{t}-\int_{0}^{t} \frac{B_{T}-B_{s}}{T-s} \mathrm{~d} s \quad \text { a.s. }
$$

And $\beta_{t}$ is $F_{t}^{B} \vee \sigma\left(B_{T}\right)$-measurable if and only if

$$
\int_{0}^{t} \frac{\left|B_{T}-B_{s}\right|}{T-s} \mathrm{~d} s<\infty .
$$

Thereby we need to stop at $t_{K-1}=T-T / K$ to avoid diverge. Instead, we take $S_{T}^{*}=X_{T}$ at $t_{K}=T$ due to the model assumption.

\subsection{Behavior of $S_{t}^{n}$}

In this subsection, we discuss about the stochastic behavior of the theoretical price process $\left\{S_{t}^{n}\right\}_{t \leq T}$ without relying on the stochastic differential equation. For this aim, we see $S_{t}^{n}$ as a function of $\beta_{t}^{n}, \beta_{s_{t}}^{n *}$ and use (6) and (7) to separate the pricing formula into the part depending on $\beta_{t}^{n}, \beta_{s_{t}}^{n *}$, and the independent part. Equation (12) can be rewritten as

$$
\begin{gathered}
S_{t}^{n}=\sum_{i \in\{0,1\}} x_{i} \bar{\pi}_{t}^{n}\left(x_{i}\right), \\
\bar{\pi}_{t}^{n}\left(x_{i}\right) \stackrel{\text { def }}{=} \frac{p_{i} \exp \left(f\left(x_{i}, X_{T}\right)+\left(g_{t} \beta_{t}^{n}+h_{s} \beta_{s}^{n *}\right) x_{i}\right)}{\sum_{i \in\{0,1\}} p_{i} \exp \left(f\left(x_{i}, X_{T}\right)+\left(g_{t} \beta_{t}^{n}+h_{s} \beta_{s}^{n *}\right) x_{i}\right)},
\end{gathered}
$$

where, for simplicity we use $s$ to denote $s_{t}$ and defined

$$
\begin{gathered}
f\left(x, X_{T}\right) \stackrel{\text { def }}{=} \kappa_{t}\left(\sigma_{n}^{2} t x X_{T}-\frac{\sigma_{n}^{2} t x^{2}}{2}\right)+\rho s \kappa_{s} \sigma_{n} \sigma_{n *}\left(x^{2}-2 x X_{T}\right) \\
+\kappa_{s}\left(\sigma_{n *}^{2} s x X_{T}-\frac{\sigma_{n *}^{2} s x^{2}}{2}\right), \\
g_{t} \stackrel{\text { def }}{=} \kappa_{t} \sigma_{n}-\kappa_{s} \sigma_{n *} \frac{s}{t} \rho, \\
h_{s} \stackrel{\text { def }}{=} \kappa_{s}\left(\sigma_{n *}-\sigma_{n} \rho\right) .
\end{gathered}
$$

We note that $f\left(x, X_{T}\right), g_{t}, h_{s}$ are independent from $\beta_{t}^{n}, \beta_{s}^{n *}$. Suppose that $\beta_{t}^{n}=\beta_{s_{t}}^{n *}=0$ for all $t \in[0, T)$ and $s_{t}<t$, then the deterministic curve $t \mapsto \bar{S}_{t}^{n}$ given by

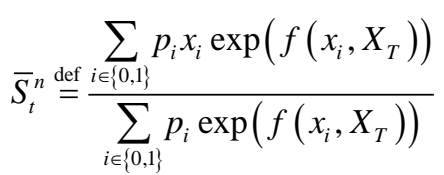

indicates the trajectory without any noise. We call this curve base line with the 
intention to represent the regression level for long-term. Figure 1 illustrates the shape of the base line in case of $X_{T}(\omega)=x_{1}=1$ and Figure 2 illustrates the case of $X_{T}(\omega)=x_{0}=0.2$. Here the other parameters are set to $\sigma_{n}=\sigma_{n *}=1, \rho=0$.

Remark 4. Since $\bar{\pi}_{t}^{n}\left(x_{0}\right)+\bar{\pi}_{t}^{n}\left(x_{1}\right)=1$, we see that $x_{0} \leq S_{t}^{n} \leq x_{1}$ almost surely. Therefore the initial information $\mathbb{P}\left(X_{T}=x_{0}\right)=p_{0}$ has great importance in case of the simplest model, i.e., $X_{T}$ takes value either $x_{0}$ or $x_{1}$.

Next, we consider the effects of $\beta_{t}^{n}$ and $\beta_{s}^{n *}$. If $g_{t} \beta_{t}^{n}+h_{s} \beta_{s}^{n *}>0$ then the theoretical price $S_{t}^{n}$ remains to fluctuate over the base line, while if $g_{t} \beta_{t}^{n}+h_{s} \beta_{s}^{n *}<0$ then $S_{t}^{n}$ remains to fluctuate below the base line. This is true regardless of the sign of $f\left(x, X_{T}\right)$. By definition, $s_{t}<t$ and then $\kappa_{s}<\kappa_{t}$, which leads to the fact that $g_{t}>h_{s}$ conditional to $\sigma_{n}=\sigma_{n *}$. Therefore, $S_{t}^{n}$ is affected by her own pure noise $\beta_{t}^{n}$ rather than $\beta_{s}^{n *}$, which would be appropriate from qualitative point of view.

The following two figures show the single trajectory of $S_{t}^{n}$ and corresponding base line given the common simulated paths $\beta_{t}^{n}, \beta_{t}^{n *}$ in case of $X_{T}(\omega)=x_{1}$ (Figure 3) and for $X_{T}(\omega)=x_{0}$ (Figure 4) respectively.

At first glance, it seems that $S_{t}^{n}$ fluctuate in conjunction with $\beta_{t}^{n}$. However, because of $\rho>0$, it is not always true that only $\beta_{t}^{n}$ determines the behavior of $S_{t}^{n}$. To see this,

$$
\begin{aligned}
& \frac{\partial}{\partial z^{1}} \mathbb{S}\left(z^{1}, z^{2} ; \sigma_{n} \sigma_{n^{*}}, t, s\right)=\sum_{i=0}^{1} x_{i} \frac{\partial}{\partial z^{1}} \bar{\pi}^{n}\left(x_{i} \mid z^{1}, z^{2}\right) \\
& =\left(\kappa_{t} \sigma_{n}-\rho s \kappa_{s} \frac{\sigma_{n^{*}}}{t}\right) \operatorname{Var}\left[X_{T} \mid \xi_{t}^{1}=z^{1}, \xi_{s}^{2}=z^{2}\right]
\end{aligned}
$$

then, from $\operatorname{Var}\left[X_{T} \mid \xi_{t}^{1}=z^{1}, \xi_{s_{t}}^{2}=z^{2}\right]>0$, we see that $\kappa_{t} \sigma_{n}-\rho s \kappa_{s} \frac{\sigma_{n *}}{t}$ determines the sign of $\frac{\partial}{\partial z^{1}} \mathbb{S}\left(z^{1}, z^{2}\right)$. If $\sigma_{n}=\sigma_{n^{*}}$, then $\kappa_{t} \sigma_{n}-\rho s \kappa_{s} \frac{\sigma_{n *}}{t}>0$ and then $S_{t}^{n}=\mathbb{S}\left(\xi_{t}^{n}, \xi_{t}^{n *} ; t, s\right)$ is monotone increasing with respect to $\xi_{t}^{n}$. However, we should note that if $\sigma_{n}<\sigma_{n *}$ then $\kappa_{t} \sigma_{n}-\rho s \kappa_{s} \frac{\sigma_{n *}}{t}$ may happen to be negative, saying that $S_{t}^{n}$ increases even if both $\beta_{t}^{n}, \beta_{s_{t}}^{n *}$ decrease.

\subsection{The Base Line and $\sigma_{n}$}

We also have an interest in parameter dependences of the base line. Figure 5 depicts the behavior of the base line as we change the parameter $\sigma=0.2,0.4, \cdots, 2.0$ while keeping $\sigma_{n}=: \sigma$ for all $n \in \mathcal{N}$ in case of $X_{T}(\omega)=x_{1}$. We can see that the larger $\sigma$ increases the quality of the market information and then the theoretical price reach its true value of $X_{T}$ more rapidly. On the other hand, Figure 6 depicts the case of $X_{T}(\omega)=x_{0}$. The smaller $\sigma$ corresponds to the less informed agent and then such an agent can not realize the default scenario until just before the default. These trend become conspicuous with the decreasing of $\sigma$. 


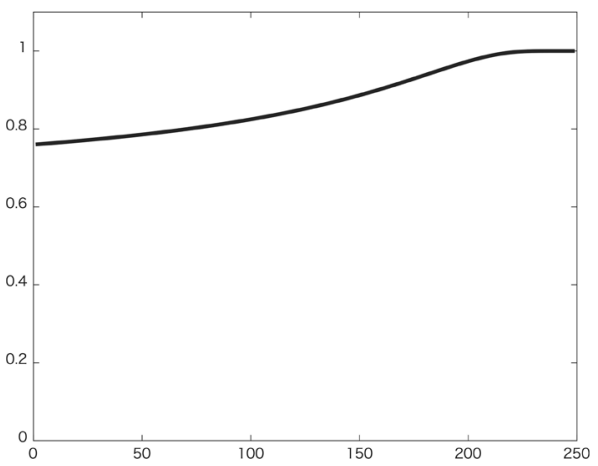

Figure 1. Base line: $X_{T}(\omega)=x_{1}=1$.

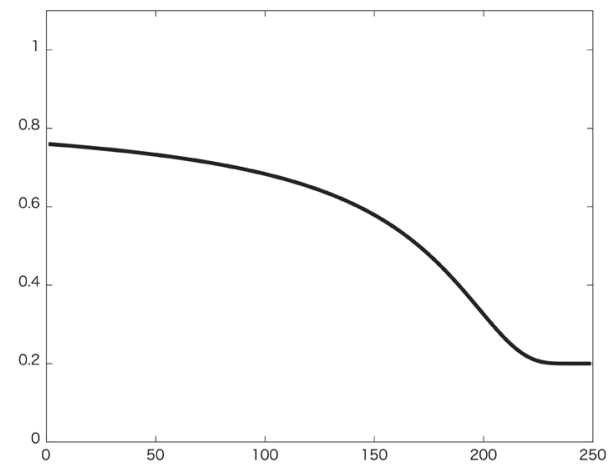

Figure 2. Base line: $X_{T}(\omega)=x_{0}=0.2$.

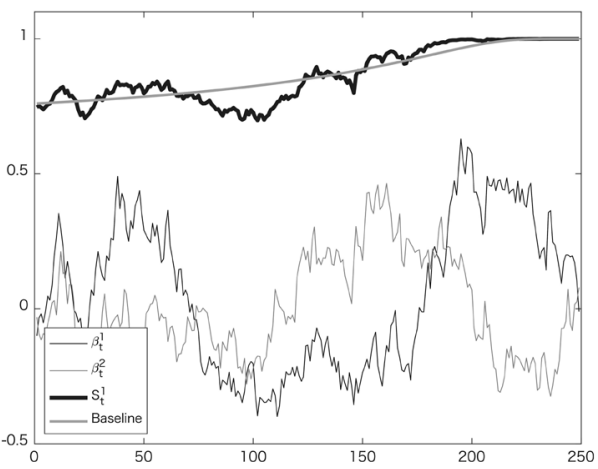

Figure 3. $X_{T}(\omega)=x_{1}$.

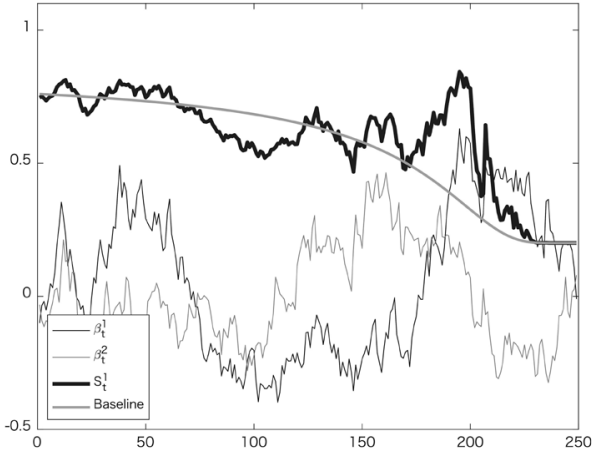

Figure 4. $X_{T}(\omega)=x_{0}$. 


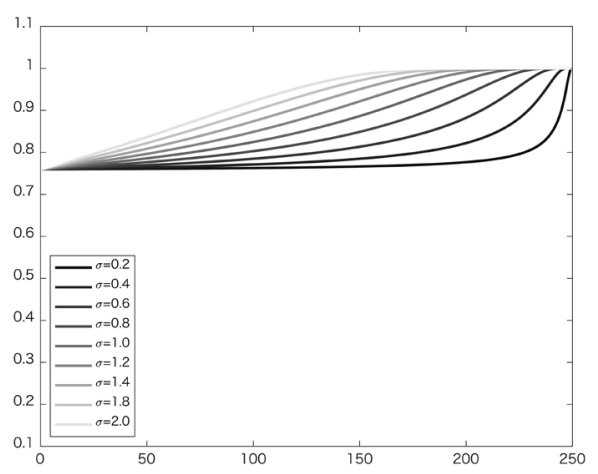

Figure 5. Base line: $X_{T}(\omega)=x_{1}$.

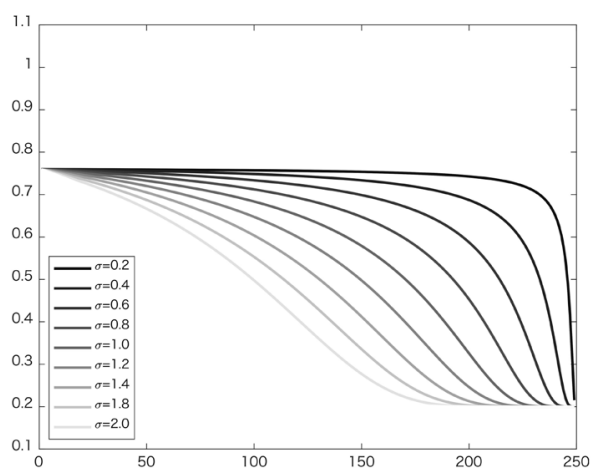

Figure 6. Base line: $X_{T}(\omega)=x_{0}$.

\subsection{The Base Line and $\rho$}

Figure 7 (Figure 8) depicts the shape of the base line, under the condition $X_{T}(\omega)=x_{1} \quad\left(X_{T}(\omega)=x_{0}\right.$ respectively $)$, as the parameter $\rho$ changing from 0 to 0.9 with 0.1 increments. We can observe that the smaller $\rho$ enables agents to reach the true value of $X_{T}$ more quickly.

It can be seen that how the shape of the base line depends on the noise correlation $\rho$ as follows. Letting $\beta_{t}^{n}=\beta_{s_{t}}^{n *}=0$ in $\pi_{t}^{n}\left(x_{0}\right)$ and $\pi_{t}^{n}\left(x_{1}\right)$, define the distribution $\bar{\pi}_{t}^{n}\left(x_{i}\right), i=0,1$ as

$$
\bar{\pi}_{t}^{n}\left(x_{i}\right)=\frac{p_{0} \exp \left(f\left(x_{i}, X_{T}\right)\right)}{p_{0} \exp \left(f\left(x_{i}, X_{T}\right)\right)+p_{1} \exp \left(f\left(x_{i}, X_{T}\right)\right)} .
$$

Then the partial differential with respect to $\rho$ leads to

$$
\begin{aligned}
& \frac{\partial \bar{\pi}_{t}^{n}\left(x_{0}\right)}{\partial \rho} \\
& =\frac{s \kappa_{s} \sigma_{n} \sigma_{n^{*}}\left(x_{0}^{2}-x_{1}^{2}-2 x_{0} X_{T}+2 x_{1} X_{T}\right) p_{0} p_{1} \exp \left(f\left(x_{0}, X_{T}\right)+f\left(x_{1}, X_{T}\right)\right)}{\left[p_{0} \exp \left(f\left(x_{0}, X_{T}\right)\right)+p_{1} \exp \left(f\left(x_{0}, X_{T}\right)\right)\right]^{2}} .
\end{aligned}
$$

Because of $s \kappa_{s} \sigma_{n} \sigma_{n^{*}} p_{0} p_{1} \exp \left(f\left(x_{0}, X_{T}\right)+f\left(x_{1}, X_{T}\right)\right)>0$, the sign of $\frac{\partial \bar{\pi}_{t}^{n}\left(x_{0}\right)}{\partial \rho}$ coincides that of $\left(x_{0}^{2}-x_{1}^{2}-2 x_{0} X_{T}+2 x_{1} X_{T}\right)=\left(x_{0}+x_{1}-2 X_{T}\right)\left(x_{0}-x_{1}\right)$. By definition, $x_{0}-x_{1}<0$, then we can conclude 


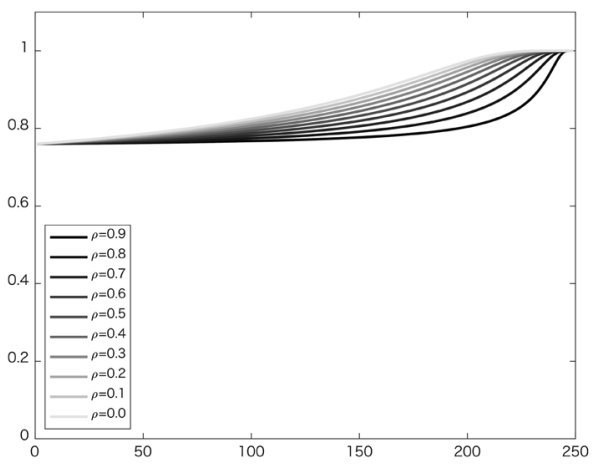

Figure 7. Base line: $X_{T}(\omega)=x_{1}$.

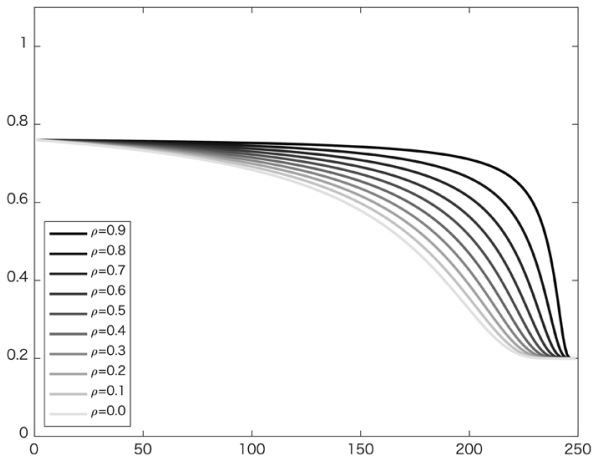

Figure 8. Base line: $X_{T}(\omega)=x_{0}$.

- in case of $X_{T}(\omega)=x_{0}, x_{0}+x_{1}-2 X_{T}=x_{1}-x_{0}>0$ hence $\frac{\partial \bar{\pi}_{t}^{n}\left(x_{0}\right)}{\partial \rho}>0$.

So if $\rho \nearrow 1$ then $\bar{\pi}_{t}^{n}\left(x_{0}\right) \searrow 0$ and $\bar{\pi}_{t}^{n}\left(x_{1}\right)=1-\bar{\pi}_{t}^{n}\left(x_{0}\right) \nearrow 1$.

- in case of $X_{T}(\omega)=x_{1}, x_{0}+x_{1}-2 X_{T}=x_{0}-x_{1}<0$ hence $\frac{\partial \bar{\pi}_{t}^{1}\left(x_{0}\right)}{\partial \rho}<0$.

So, if $\rho \nearrow 1$ then $\bar{\pi}_{t}^{n}\left(x_{0}\right) \nearrow 1$ and $\bar{\pi}_{t}^{n}\left(x_{1}\right)=1-\bar{\pi}_{t}^{n}\left(x_{0}\right) \searrow 0$.

\subsection{Behavior of the Transaction Price $S_{t}^{*}$}

Now we turn our interests from the theoretical price of a single agent to the transaction price (market price) determined from all submitted orders in accordance with the matching rule as in Assumption 3. Let us show four examples (Figures 9-12) depicting 20 sample paths of transaction price $S_{t}^{*}$ for each, with changing parameters as $(\rho, N)=(0,2),(0,100),(0.9,2),(0.9,100)$ respectively. For better(easy) understanding for the situation $N \rightarrow+\infty$ later, we assumed $\sigma_{i}=1$ for all $i \in \mathcal{N}$. Figure 9, where $\rho=0$ and $N=2$ are chosen, indicates the path fluctuating wide range of the interval $\left[x_{0}, x_{1}\right]=[0.2,1]$. This would come from the sequential directionless transactions due to mutually independent noise.

Changing from $N=2$ to $N=100$ while keeping $\rho=0$ makes a significant difference as depicted in Figure 9 and Figure 10. First, we remember that all the agents can access to overall market information flow rate $\bar{\sigma}$ defined as a 


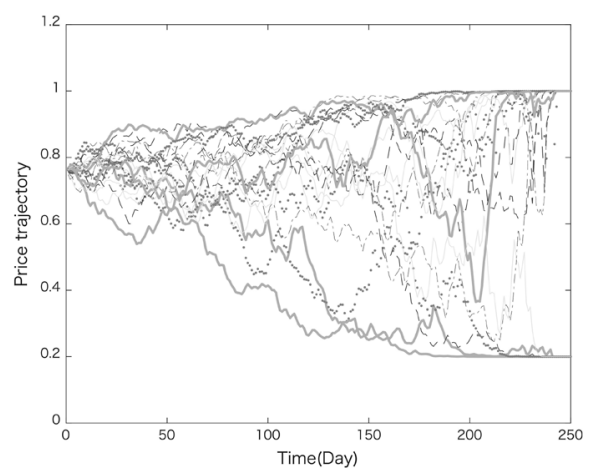

Figure 9. $S_{t}^{*}(\rho=0, N=2)$.

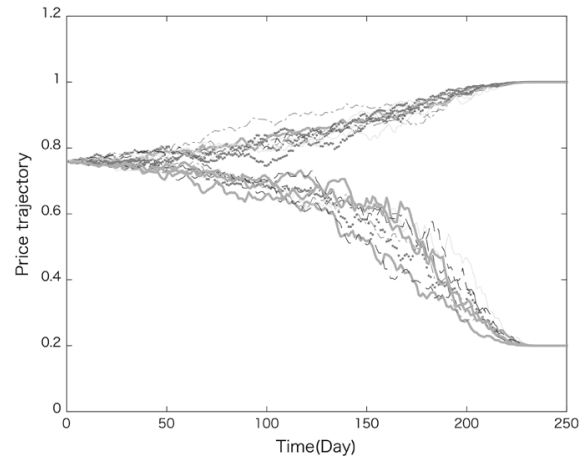

Figure 10. $S_{t}^{*}(\rho=0, N=100)$.

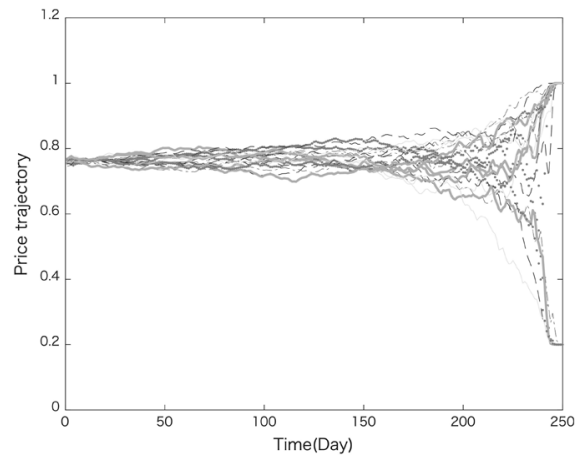

Figure 11. $S_{t}^{*}(\rho=0.9, N=2)$.

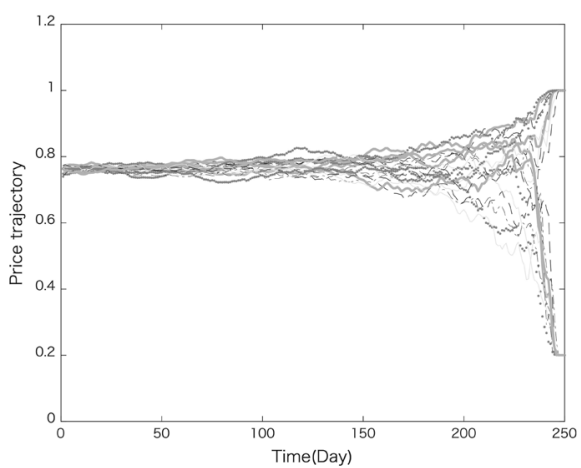

Figure 12. $S_{t}^{*}(\rho=0.9, N=100)$. 
median of all agents' $\sigma$, and that the pricing formula (11) or (12) is an increasing function of $\beta_{t}^{i}$ (hence of $\xi_{t}^{i}$ too). Therefore, at the stage of the information update (13), supplemental information $\xi_{t}^{n *}$ calculated via inverse operation from $S_{t}^{*}$ (median of $\left\{S_{t}^{i}\right\}_{i \in \mathcal{N}}$ ) would be also a median of all $\left\{\xi_{t}^{i}\right\}_{i \in \mathcal{N}}$.

When we increase $N$, "what will happen" would be understood by an analogy of the central limit theorem. Here, for an intuitive understanding, we refer so-called the Central Limit Theorem for Medians as stated below.

Theorem 1 (Central Limit Theorem for Medians). Let $X_{1}, X_{2}, \cdots, X_{n}$ be i.i.d. random variables with probability distribution function $f(x)$ and cumulative distribution function $F(x)$. If $f(0)=\frac{1}{2}$, and $f$ is continuous at 0 , then the sampling distribution of the median $M$ is approximately normal. More precisely, $\sqrt{n} M \stackrel{d}{\longrightarrow} N\left(0, \frac{1}{4 f(0)^{2}}\right)$ as $n \rightarrow+\infty$.

The proof is based on the property of the order statistics and technical use of Stirling's formula, and it is provided, for example, in Chapter 1 of Steven J. Miller [13].

If $N \rightarrow+\infty$, the median of all $\left\{\xi_{t}^{i}\right\}_{i \in \mathcal{N}}$ get close to $\sigma_{i} t X_{T}=t X_{T}$. This is nothing but the point on the base line $t \mapsto \bar{S}_{t}^{n}$ at time $t$ determined by taking $\beta_{t}^{i}=0$. Consequently, we see if $N \rightarrow+\infty$, transaction price tends to approach the base line.

Changing from $\rho=0$ to $\rho=0.9$ while keeping $N=2$ also makes a significant difference as depicted in Figure 9 and Figure 11. The difference can be understood by considering the behavior of the base line depicted in Figure 7 and Figure 8. However, if we fix $\rho$ with a high level, trajectories of the transaction price process look almost unchanged even if $N$ increased as depicted in Figure 11 and Figure 12. This characteristic would be revisited in the next subsection in order to grasp the dynamics of $S_{t}^{*}$ in terms of its volatility.

\subsection{Volatility of $S_{t}^{*}$}

We wish to investigate the stochastic behavior of the transaction price process in terms of their volatilities. For this purpose, we run Algorithm 1 to generate $m$ samples of trajectory of the transaction price, and compute sample volatilities for each trajectory, then take expectation of those volatilities. Figure 13 depicts the sample volatility of transaction prices with $\sigma_{n}=1$ for all $n \in \mathcal{N}$ but varying $\rho$ and market size $N$.

It is interesting to see that the transaction price volatility depends on the noise correlation $\rho$ as well as the market size $N$. The simplest $N=2$ case, which has already been reported in [10], would be easy to understand. As $\rho$ increases, each agent is much more likely to receive similar market information and then their theoretical prices get closer, so the transaction price fluctuate gently.

However, the situation changes as $N$ increases. In such cases, as we mentioned 


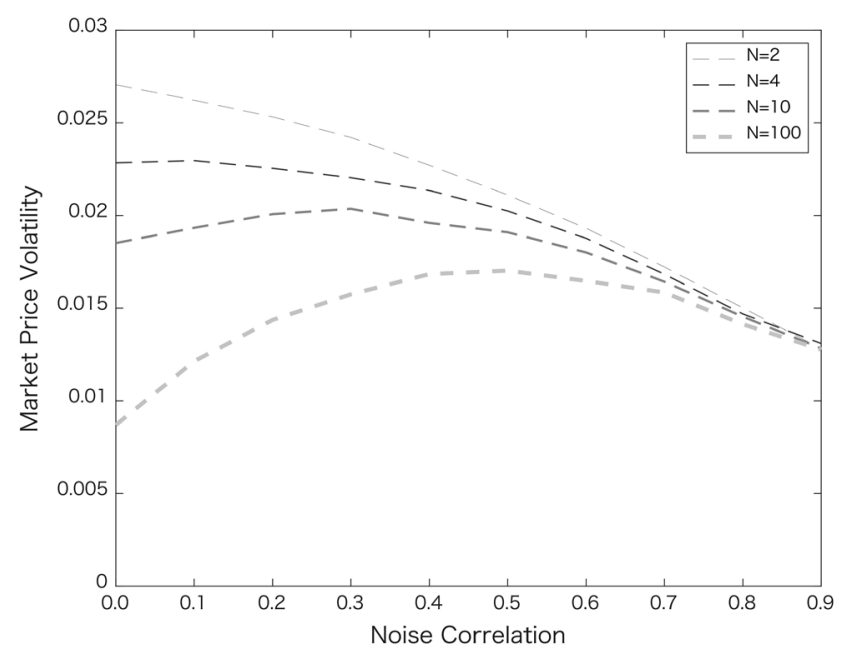

Figure 13. Transaction price volatilities as a function of $\rho$.

in the previous subsection, we see that the transaction price fluctuate fairly close to the base line (Figure 10 and Figure 12) so the volatilities become smaller. Whereas, if $\rho$ is quite large, the transaction price trajectories show a significant resemblance regardless of the level of $N$. So the right endpoints of the dashed line in Figure 13, which correspond to $\rho=0.9$, seem to pinned to fixed value 0.013 .

\section{Long Term Memory of $S_{t}^{*}$}

Since the learning procedure has been built in our framework, one may expect that the transaction price processes have a trend. This kind of behavior would be find out, for instance, by looking at the Hurst exponent. The Hurst exponent $H$ is well known statistical measure to estimate self-similarity, in other words, whether the price process has a long term memory. In particular, $H=0.5$ indicates the standard Brownian motion while $H>0.5$ indicates a trend reinforcing time series, which can be sometimes observed in real financial market, as many empirical research report.

\section{Hurst Exponent}

A lot of estimation methods for Hurst exponent are known, for example, aggregated variance method, discrete variations method, Higuchi method, Poggi and Viano method, Kurrchenko's method, and so on, as Section 9 of Prakasa Rao [14] explain, we employ here originally proposed method of Edwin Hurst [15].

Let $x_{i}:=\log S_{t_{i}}^{*}-\log S_{t_{i-1}}^{*}$ be a daily return of the transaction price generated by the simulation and set $x_{j}(i):=x_{j+i-1}$. Then we define the ratio called the rescaled adjusted range $R_{j}(\tau) / V_{j}(\tau)$ where

$$
R_{j}(\tau)=\max _{1 \leq k \leq \tau} \sum_{i=1}^{k}\left(x_{j}(i)-\left\langle x_{j}\right\rangle_{\tau}\right)-\min _{1 \leq k \leq \tau} \sum_{i=1}^{k}\left(x_{j}(i)-\left\langle x_{j}\right\rangle_{\tau}\right),
$$




$$
V_{j}(\tau)=\sqrt{\frac{1}{\tau} \sum_{i=1}^{\tau}\left(x_{j}(i)-\left\langle x_{j}\right\rangle_{\tau}\right)^{2}}
$$

and the bracket $\left\langle x_{j}\right\rangle_{\tau}$ denotes the sample mean of $\tau$ samples of $x_{j}$, that is, $\left\langle x_{j}\right\rangle_{\tau}=\left(x_{j}(1)+\cdots+x_{j}(\tau)\right) / \tau$. It is believed that the $R / V$-statistic is proportional to $\tau^{H}$ as $\tau \rightarrow \infty$ for fractional Gaussian noise. Assuming this property, we regress $\log \left\langle R_{j}(\tau) / V_{j}(\tau)\right\rangle$ against $\log \tau$ to obtain an estimator for the Hurst exponent $H$. Thus the Hurst exponent $H$ is given as a slope

$$
H:=\lim _{\tau \rightarrow \infty} \frac{\log \left\langle R_{j}(\tau) / V_{j}(\tau)\right\rangle}{\log (\tau)} .
$$

For numerical calculations, we set $T=1, K=1000$ and generated 100000 samples for $R / V$-statistic (rescaled adjusted range) to regress against $\log \tau$, where $\tau=4,8,16,32,64,128,256,512$ as shown in Table 1 . The results in the column Slope $(H)$ indicate the estimated Hurst exponent. In these results, we can see that as correlation increases Hurst exponent increases. This is intuitively acceptable because higher correlation induces similar theoretical prices among the market participants, and tend to adhere fundamentally to the previous market price. Such a feature would appear as a long-term memory of market price.

Figure 14 depicts the other cases of $N=2,4,10,100$ for different level of noise correlations. We can see that $N=100, \rho=0$ case has almost $H=0.5$, while the other cases have long memory. It is interesting to see that Hurst exponent also depends on the market size $N$, and the differences due to the market size decreases as noise correlation increases to $\rho=0.9$. We can suggest that the information based dealer model would be one of the promising candidate to model agent-driven market behaviors.

Table 1. Estimated $\log _{10}\left\langle\frac{R_{j}(\tau)}{V_{j}(\tau)}\right\rangle$ and their slopes against $\log _{10}(\tau)$ for $N=10$ agents.

\begin{tabular}{cccccccccc}
\hline & $\tau=4$ & $\tau=8$ & $\tau=16$ & $\tau=32$ & $\tau=64$ & $\tau=128$ & $\tau=256$ & $\tau=512$ & Slope $(H)$ \\
\hline $\log (\tau)$ & 0.6021 & 0.9031 & 1.2041 & 1.5051 & 1.8062 & 2.1072 & 2.4082 & 2.7092 \\
$\rho=0$ & 0.2379 & 0.4752 & 0.6849 & 0.8700 & 1.0358 & 1.1889 & 1.3340 & 1.4762 & 0.57895 \\
$\rho=0.1$ & 0.2380 & 0.4757 & 0.6862 & 0.8746 & 1.0450 & 1.2026 & 1.3536 & 1.5019 & 0.59148 \\
$\rho=0.2$ & 0.2381 & 0.4765 & 0.6885 & 0.8779 & 1.0519 & 1.2132 & 1.3677 & 1.5194 & 0.60003 \\
$\rho=0.3$ & 0.2380 & 0.4763 & 0.6891 & 0.8808 & 1.0565 & 1.2212 & 1.3787 & 1.5334 & 0.60711 \\
$\rho=0.4$ & 0.2380 & 0.4770 & 0.6901 & 0.8823 & 1.0603 & 1.2273 & 1.3877 & 1.5442 & 0.61244 \\
$\rho=0.5$ & 0.2380 & 0.4772 & 0.6912 & 0.8847 & 1.0638 & 1.2326 & 1.3943 & 1.5530 & 0.61668 \\
$\rho=0.6$ & 0.2380 & 0.4774 & 0.6916 & 0.8866 & 1.0668 & 1.2364 & 1.3992 & 1.5597 & 0.61990 \\
$\rho=0.7$ & 0.2382 & 0.4772 & 0.6920 & 0.8875 & 1.0687 & 1.2398 & 1.4042 & 1.5667 & 0.62321 \\
$\rho=0.8$ & 0.2382 & 0.4775 & 0.6928 & 0.8893 & 1.0708 & 1.2427 & 1.4078 & 1.5717 & 0.62554 \\
$\rho=0.9$ & 0.2381 & 0.4777 & 0.6932 & 0.8890 & 1.0716 & 1.2445 & 1.4115 & 1.5767 & 0.62783 \\
\hline
\end{tabular}




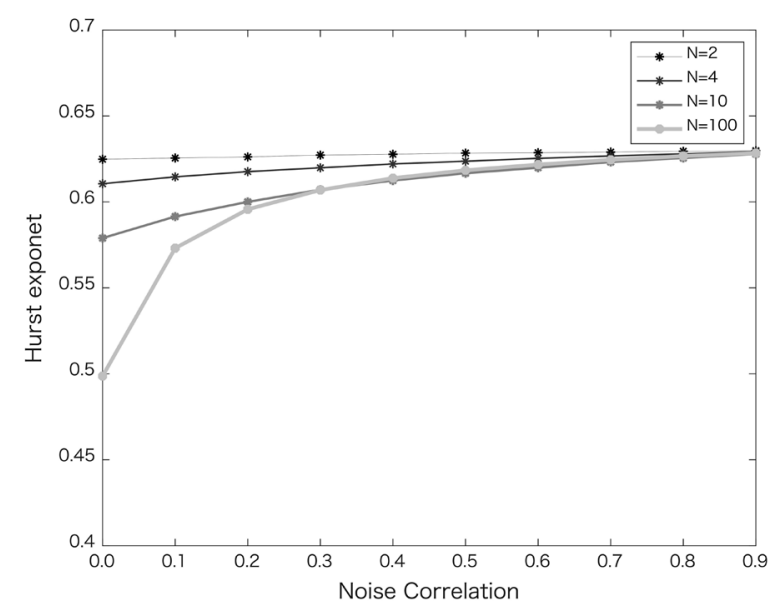

Figure 14. Hurst exponents as a function of the noise correlation for different size of $N$.

\section{Conclusion}

We considered the dealer model under information-based approach to investigate the stochastic behavior of the transaction price. We extended the two-agent based dealer model proposed by Nadi Serhan Aydin to the multi-agent model so as to study the effect of the market size. For this purpose, we introduced simple matching role to determine the transaction price among the submitted prices. Possibilities of the other matching roles, and considering bid-ask spread are left to further research. The contributions of the paper are twofold. Firstly, we paid attention to the noise correlation and the market size that controls the price process volatility. The results were intuitively acceptable and then suggesting that we cannot ignore the interaction of the noise. Secondly, because of the information updating procedure, we found numerically that the price process possesses the long-term memory, one of the major experimental findings of the financial markets. Thus, we showed potential of the information-based approach to deal with self-similar price processes under some simple assumptions. Unfortunately, it is not easy to derive stochastic differential equation for transaction price, analysis is now limited to the Monte Carlo simulations. Further research would be needed to derive the stochastic differential equation driven by a fractional Brownian motion, especially the volatility function in terms of the noise correlation and the market size.

\section{Acknowledgements}

We thank Dorje C. Brody and Nadi Serhan Aydın for helpful suggestions. This work was supported by the Research Institute for Mathematical Sciences (RIMS), a Joint Usage/Research Center located in Kyoto University. We are grateful to participants of the FMA2018 at RIMS for valuable comments to improve our early version [10] of the study and for motivations of the current version. The authors would like to thank the editor and the referee who provided valuable suggestions on our submitted manuscript. 


\section{Conflicts of Interest}

The authors declare no conflicts of interest regarding the publication of this paper.

\section{References}

[1] Brody, D.C., Hughston, L.P. and Macrina, A. (2008) Information-Based Asset Pricing. International Journal of Theoretical and Applied Finance, 11, 107-142. https://doi.org/10.1142/S0219024908004749

[2] Macrina, A. (2006) An Information-Based Framework for Asset Pricing; X-factor theory and Its Applications. Ph.D. Thesis, University of London, arXiv:0807.2124.

[3] Hoyle, E. (2010) Information-Based Models for Finance and Insurance. Ph.D. Thesis, Imperial College London, London, arXiv:1010.0829.

[4] Rutkowski, M. and Yu, N.N. (2007) An Extension of the Brody-Hughston-Macrina Approach to Modeling of Defaultable Bonds. International Journal of Theoretical and Applied Finance, 10, 557-589. https://doi.org/10.1142/S0219024907004263

[5] Brody, D.C., Hughston, L.P. and Macrina, A. (2011) Modelling Information Flows in Financial Markets. In: Di Nunno, G. and Øksendal, B., Eds., Advanced Mathematical Methods for Finance, Springer, Berlin, Heidelberg, 133-153. https://doi.org/10.1007/978-3-642-18412-3 5

[6] Brody, D.C., Davis, M.H.A., Friedman, R.L. and Hughston, L.P. (2009) Informed Traders. Proceedings of the Royal Society A, 465, 1103-1122. https://doi.org/10.1098/rspa.2008.0465

[7] Macrina, A. and Parbhoo, P.A. (2010) Security Pricing with Information-Sensitive Discounting. In: Kijima, M., Hara, C. and Tanaka, K., Eds., Recent Advances in Financial Engineering 2009: Proceedings of the KIER-TMU International Workshop on Financial Engineering 2009, World Scientific Publishing Company, Hackensack, NJ. https://doi.org/10.1142/9789814304078 0006

[8] Ayd, N.S. (2017) Financial Modelling with Forward-Looking Information-An Intuitive Approach to Asset Pricing. Springer International Publishing, New York.

[9] Brody, D.C., Hughston, L.P. and Macrina, A. (2007) Beyond Hazard Rates: A New Framework for Credit-Risk Modelling. In: Jarrow, F. and Elliott, Y., Eds., Advances in Mathematical Finance, Birkhäuser, Boston, MA, 231-257. https://doi.org/10.1007/978-0-8176-4545-8_ 13

[10] Kondo, K., Takada, H. and Fukuda, K. (2019) Information-Based Approach for Dealer Model (In Japanese). RIMS Kokyuroku 2111, Research Institute for Mathematical Sciences, Kyoto University, Kyoto, Japan, 45-64.

[11] Kloeden, P.E. and Platen, E. (2011) Numerical Solution of Stochastic Differential Equations. In: Stochastic Modelling and Applied Probability, Volume 23, Springer-Verlag, Berlin, Heidelberg.

[12] Glasserman, P. (2003) Monte Carlo Methods in Financial Engineering. In: Stochastic Modelling and Applied Probability, Springer-Verlag, New York. https://doi.org/10.1007/978-0-387-21617-1

[13] Miller, S.J. (2017) The Probability Lifesaver: All the Tools You Need to Understand Chance. Princeton University Press, Princeton, NJ. https://doi.org/10.1515/9781400885381

[14] Prakasa Rao, B.L.S. (2010) Statistical Inference for Fractional Diffusion Processes, Wiley Series in Probability and Statistics. John Wiley \& Sons Ltd., New York.

[15] Hurst, H.E. (1951) Long-Term Storage Capacity of Reservoirs. Transactions of American Society of Civil Engineers, 116, 770-808. 